\title{
Intercambio, guerra y venganza: el lanceamiento de Ompore Omehuai y su esposa Buganei Caiga
}

Roberto Narváez

\begin{abstract}
RESUMEN
Los grupos familiares de pUeblos en aislamiento QUe habitan en la Región del Yasuní, Mantienen DINÁMICAS SOCIALES Y CULTURALES PROPIAS EN UN REMANENTE DEL TERRITORIO DE USO Y MOVILIDAD TRADICIONAL QUE COMPARTEN CON VARIOS POBLADOS WAORANI**. ESTE ESPACIO COLINDA CON ACTORES EXTERNOS QUE SE HAN ASENTADO Y QUE EJERCEN PRESIÓN SOBRE EL MISMO, GENERANDO CONFLICTOS, MANIFESTADOS EN EVENTOS DE VIOLENCIA. En 2013, un ATAQUe CON laNZAS TERMinó CON LA VIDA DE UNA PAREJA de ANCIANOS WAORANI. EL HOMBRE LANCEADO ERA UN LÍDER TRADICIONAL WAORANI QUE HABÍA VUELTO A VIVIR EN EL TERRITORIO DE SUS ANTEPASADOS, COMPARTIÉNDOLO CON GRUPOS FAMILIARES EN AISLAMIENTO, LUEGO DEL CONTACTO QUE VIVIÓ DESDE 1970. A pesar de las particularidades de este ataque, desde la sociedad nacional no SE buSCó SU REAL Comprensión; más allá del proceso Judicial que inició la Fiscalía General del Estado, a través de la Comisión de la Verdad y Derechos Humanos, para identificar aspectos culturales Que puedan dar una GUÍA SOBRE ESTAS MANIFESTACIONES DE VIOLENCIA.
\end{abstract}

Palabras claves: pueblos en aislamiento - intercambio - guerra - Venganza.

Abstract

The isolated family groups that live in the Yasuní Region Maintain their own SOcial ANd Cultural DYNAMICS WITHIN A REMNANT OF THEIR TRADITIONAL TERRITORY OF USE AND MOBILITY WHICH IS SHARED WITH

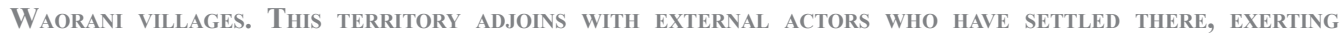
PRESSURE IT, GENERATING CONFLICTS WHICH MANIFEST IN VIOLENT EVENTS. IN 2003 A SPEAR ATTACK ENDED WITH A

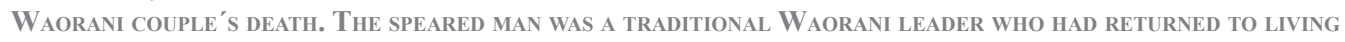
IN HIS ANCESTRAL TERRITORY, SHARING WITH ISOLATED FAMILIES AFTER HAVING LIVED IN CONTACT SINCE THE 1970 'S.

* Licenciado en Antropología por la Pontificia Universidad Católica del Ecuador con especialización en derechos humanos y pueblos indígenas. Perito antropólogo acreditado por el Consejo de la Judicatura y designado por la Fiscalía General del Estado en casos de pueblos en aislamiento e interculturalidad. Correo electrónico: robertonarvaezc@gmail.com.

Los datos del presente artículo se obtuvieron a partir de entrevistas directas a los familiares de las víctimas, entre mayo y septiembre de 2013, mientras participé como perito antropólogo para la Fiscalía General del Estado durante el proceso de indagación previa, diligencia de reconocimiento del lugar de los hechos y el proceso judicial que hasta la fecha no concluye. El trabajo de campo incluyó visitas y entrevistas a diversos informantes en los poblados waorani de Yarentaro, Dicaro, Ganquetapade, Guiyero, Peneno, Yawepade, Baameno, y Boanamo, además de varias entrevistas que fueron realizadas en la ciudad del Coca.

** A pesar de que el proceso de contacto con los waorani inicio en la década del 60 del siglo anterior, se considera un pueblo de reciente contacto, con grandes limitaciones en cuanto a su conocimiento del Estado y sus instituciones, producto de una relación inequitativa y en muchos casos agresiva desde la sociedad envolvente. 
IN SPITE OF THE PARTICULARS OF THIS ATTACK, A TRUE UNDERSTANDING WAS NOT INVESTIGATED BY THE ECUADORIAN national society, other than the Judicial process started by The State Attorney General through the Commission for Truth and Human Rights, to identify CUltural aspects Which Can provide a GUIDE To UNDERSTANDING THESE VIOLENT MANIFESTATIONS.

KEYWORDS: ISOLATED INDIGENOUS PEOPLE - EXCHANGE - WAR - REVANGE.

\section{Introducción}

$\mathrm{E}$ n marzo de 2013 una pareja de ancianos waorani, Ompore Omehuai y su esposa Buganei Caiga, pertenecientes al grupo conocido como Wepeiri (Cabodevilla, 1999; Labaka, 1988; Trujillo, 2011) fueron lanceados ${ }^{1}$ y muertos por un grupo de individuos de familias en aislamiento en la región del Yasuní ${ }^{2}$. El ataque se produjo a menos de cien metros del poblado waorani de Yarentaro, ubicado dentro del bloque petrolero 15 operado por la empresa Repsol - YPF en la parroquia Cononaco, cantón Aguarico de la provincia de Orellana, Amazonía del Ecuador.

El lanceamiento dejó abiertas una serie de interrogantes sobre las causas que motivaron este hecho, principalmente porque Ompore había mantenido varios encuentros ${ }^{3}$ con grupos familiares en aislamiento en el territorio de interrelación que comparten. Para los familiares de las víctimas el ataque generó inquietud, ya que retornó el escenario de guerra después de un prolongado periodo de paz desde la incorporación de los grupos familiares Wepeiri al contacto a finales de la década de los 70 del siglo anterior;; y reavivó los referentes culturales donde la muerte desencadena un tiempo de guerra incitado por la venganza.

La reacción de las familias elevó el nivel de conflictividad que de por sí ya existe en la región del Yasuní; para empezar, se buscó soporte en las instituciones públicas que nunca atendieron los ofrecimientos realizados, tanto para velar por la seguridad de las familias de Yarentaro y Dicaro ante posibles nuevos ataques, como también con medidas de reparación por la muerte de los ancianos. Así, las familias buscaron condiciones de autodefensa, como prepararse con armas de fuego y comprar municiones. Por otra parte, en el exterior (en el mundo no waorani), la opinión pública nacional y las acciones estatales expusieron una falta de información y desconocimiento sobre los pueblos en aislamiento así como las dinámicas profundas y persistentes en un territorio donde se desenvuelven además los waorani como pueblo de reciente contacto. Las acciones del Estado se manejaron con un discurso de "violencia natural" de los waorani y de los grupos familiares en aislamiento, generalizando y "entendiendo" como un conflicto abierto entre ambos grupos, sin comprender la persistencia de diferentes grupos familiares tanto waorani como de grupos familiares de pueblos en aislamiento.

El análisis que se presenta a continuación expone varias particularidades en torno a las relaciones en la Amazonía, entre las cuales el intercambio y la circulación de bienes constituyen hechos sociales en un entorno ambiental de foresta tropical, y espacio de dinámica constante entre naturaleza y cultura. Se expondrá información para comprender las dinámicas de circulación de bienes en los grupos familiares en aislamiento, y la posible limitación de dicha circulación como origen de una etapa de violencia, que pudo haberse materializado en el ataque contra los ancianos Ompore y Buganei. Así, la información nos dará líneas para

1 Muerte provocada con lanzas tradicionales.

2 El Yasuní comprende la bioregión caracterizada por una alta biodiversidad en la selva tropical ecuatoriana ubicada entre los ríos Napo (al norte) y Curaray (al sur).

3 Durante la investigación, los familiares de Ompore y pobladores de Yarentaro narraron encuentros pacíficos con cierto nivel de tensión.

4 El territorio donde se desarrollaron estos hechos comprende el sector de Ahuemuro (entre los ríos Tiputini y Yarentaro), cuyas familias (Wepeiri) fueron contactadas por misioneros capuchinos a finales de los 70 (Labaka, 1988), en un proceso donde la actividad petrolera había despuntado hacia el sur del río Napo. 


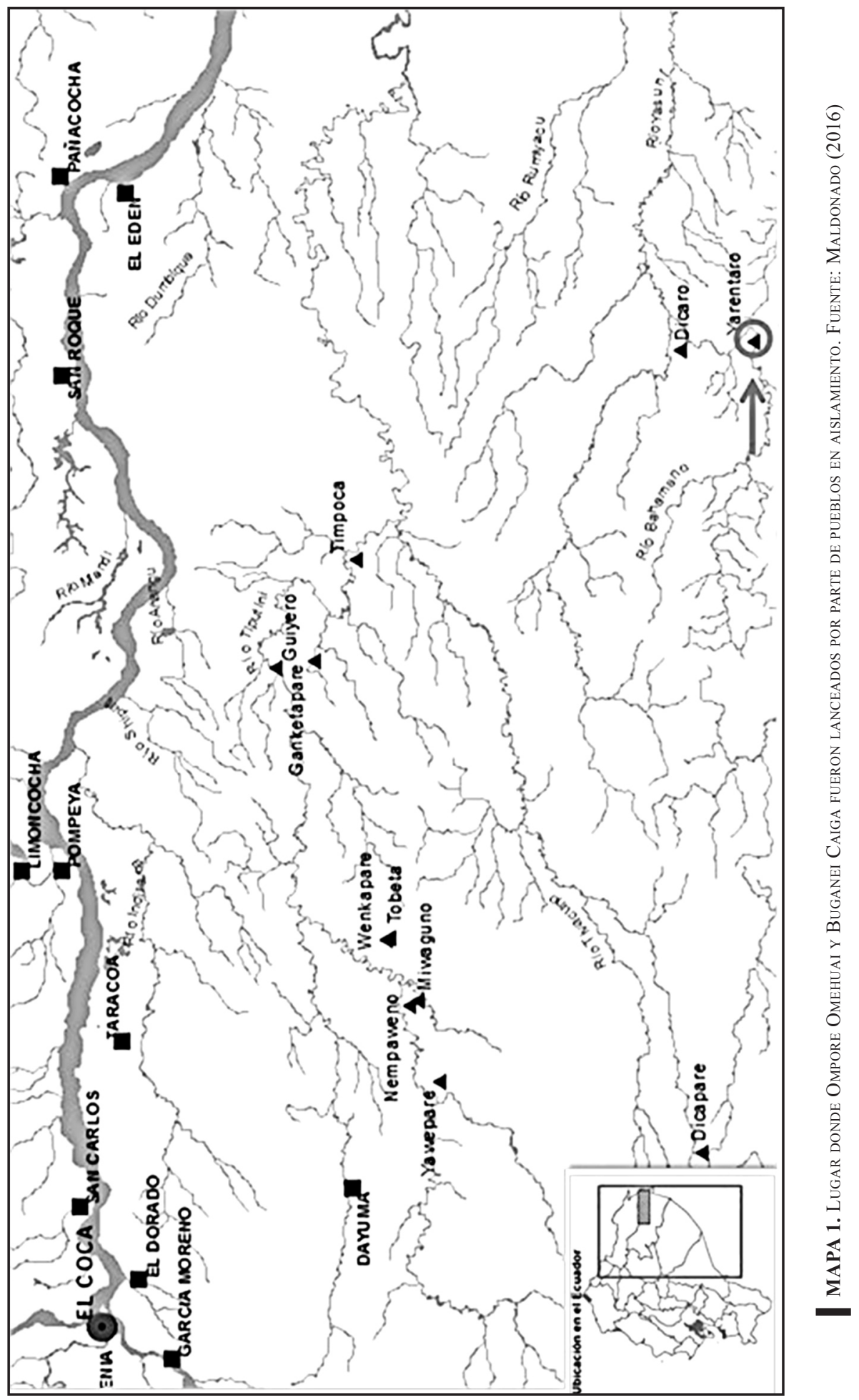


identificar varios de los elementos que motivaron la violencia en los grupos familiares en aislamiento en el Yasuní, y aportar a una mayor comprensión de la realidad socio cultural de esta región de la Amazonía ecuatoriana.

Para el desarrollo de los contenidos se iniciará con una exposición acerca de los grupos familiares en aislamiento que habitan en el Yasuní. A continuación, y utilizando información etnográfica, se caracterizarán las dinámicas de los grupos familiares en aislamiento y las relaciones que existen con pobladores waorani. En el capítulo final se analizaran las posibles causas del ataque y la situación en la que se encuentran los grupos familiares en aislamiento en la región del Yasuní.

\section{Los pueblos en aislamiento de la región del Yasuní}

Pueblos indígenas aislados (PIA) o en situación de aislamiento son denominación que se da a aquellos grupos familiares que guardan sus formas de vida tradicional y que han optado por mantenerse al margen de las sociedades nacionales (Rivas, 2003). Naciones Unidas define a los pueblos indígenas en aislamiento como "pueblos o segmentos de pueblos indígenas que no mantienen contactos regulares con la población mayoritaria y que además suelen rehuir todo tipo de contacto con personas ajenas a su grupo (Oficina del Alto Comisionado de Naciones Unidas para Derechos Humanos, 2012: 8).

En el caso de Ecuador, los pueblos en aislamiento del Yasuní ${ }^{5}$ son grupos familiares waorani ${ }^{6}$ escindidos en varios períodos históricos, que se mantuvieron y mantienen en aislamiento en el territorio ubicado entre el Tiputini y Curaray, entre las actuales vías Auca y Maxus, conservando su forma de vida tradicional (Narváez, 2013a). Su organización se da alrededor de grupos familiares residenciales denominadas nanicabo que están bajo el liderazgo de un miembro masculino reconocido, y quien es percibido por el grupo como el proveedor, cazador, recolector o guerrero que garantiza la seguridad y supervivencia del nanicabo (Rival, 1996). Los grupos familiares ocupan un territorio endógamo amplio conformando un segmento regional en el cual la residencia tiene patrones establecidos por línea materna ${ }^{7}$. Durante el proceso de contacto que inició a finales de la década del 60 del siglo anterior, y estuvo a cargo de misioneros evangélicos, varios nanicabo waorani fueron reducidos al poblado de Teweno (principalmente nanicabos de los grupos Guikitairi, Baihuairi y Nihuairi - Piyemoiri), mientras que varios nanicabo Wepeiri y Nihuairi - Piyemoiri permanecieron según su forma de vida en su territorio tradicional.

Posteriormente, y por la presión petrolera en los años 70, misioneros católicos del Vicariato del Aguarico iniciaron un proceso de contacto con varios de esos nanicabo que se habían mantenido en aislamiento, integrando a los nanicabos Wepeiri, más no a los Nihuairi - Piyemoiri. De la información histórica y etnográfica (Cabodevilla, 1999; Labaka , 1988; Rival, 1996; Trujillo, 2011) se conoce que del grupo Nihuairi - Piyemoiri varios nanicabo se mantuvieron sin contacto, entre ellos los Tagaeri, además de un grupo reconocido por los waorani como Taromenane. En entrevistas recientes a ancianos del poblado waorani de Baameno, se conoció de la existencia de un nanicabo Dagakairi cuyo territorio tradicional se ubica entre los ríos Cononaco y Nashiño (Entrevista a Kemperi, mayo 2016, Baameno). Así, los grupos familiares en aislamiento que se desenvuelven en la región del Yasuní en la actualidad son nanicabos waorani sin contacto.

5 Existen importantes indicios de presencia de otros grupos en aislamiento en el territorio al sur del río Curaray, donde varias comunidades sapara y kichwa identifican una zona de vida y un activo corredor de tránsito de pueblos en aislamiento entre los ríos Curaray, Tigre y Bobonaza (Narváez, 2014), donde se identifica una filiación cultural diferente a la waorani cuya información es necesario profundizar.

6 Se ha generalizado la denominación de pueblos en aislamiento a Tagueiri, Taromenane, Wiñatari y Oñamenani; sin embargo, y a partir de información etnográfica planteamos la existencia de varios grupos familiares en aislamiento que se han escindido de un tronco cultural waorani en varios períodos históricos.

7 En la práctica, la pareja recién casada se establecerá en el territorio de la familia de la mujer. Por ello, en cada casa pueden habitar los padres mayores, las/os hijas/os solteros, las hijas casadas con sus maridos e hijos, aproximadamente unas 30 personas. 


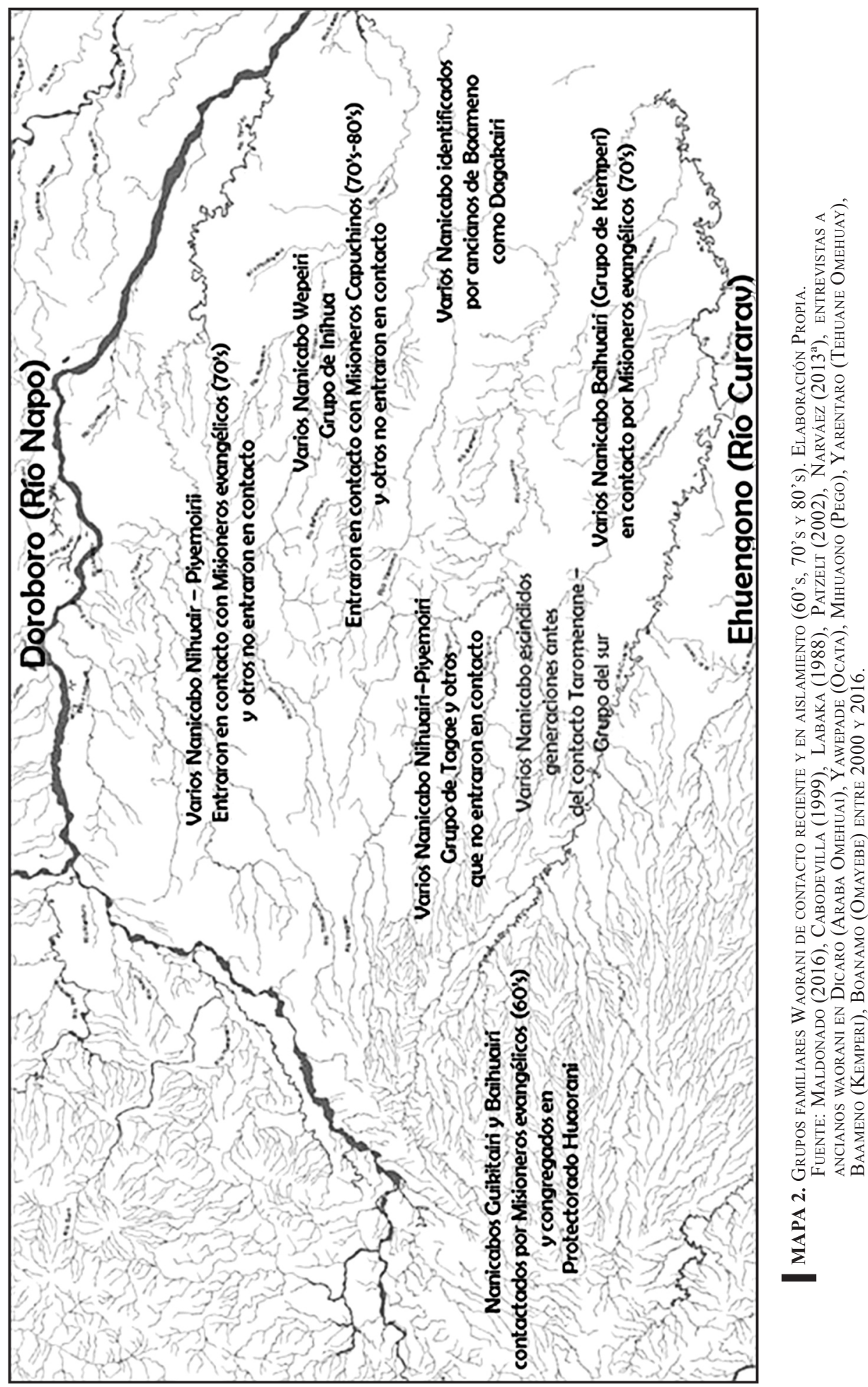


La organización social de los grupos familiares waorani en aislamiento sigue el patrón de modelo residencial donde se da una distribución en su territorio tradicional; esto es una casa típica construida con una arquitectura tradicional y denominada en wao tededo como onko ${ }^{8}$, en el que habitaba el nanicabo compuesto por entre treinta y cuarenta personas (Rival, 1996; Yost, 1978; Cabodevilla, 1999). Estos grupos familiares comparten un territorio de caza y recolección, tanto con otros grupos familiares en aislamiento como con poblados waorani e incluso colonos, en varios sectores del territorio tradicional waorani, ubicado entre los ríos Napo y Curaray. La forma de subsistencia de las familias en aislamiento se basa en la recolección y cacería, y algunos espacios dispersos donde cultivan yuca y otros productos, llamados kewenkode ${ }^{9}$. Varios estudios señalan los conocimientos de la diversidad de recursos del bosque utilizados tradicionalmente por los waorani, cuya principal fuente de alimento constituyen los productos recolectados de la selva más que los cultivos de yuca (Cerón y Montalvo, 1998; Cipolleti, 2002; Tirdmarsh, 1945).

La dinámica de movilidad tradicional waorani se encuentra anclada a un territorio extenso donde la selva constituye un espacio doméstico de abundancia (Rival, 2015), en el cual los antepasados realizaron un proceso de domesticación a través de la siembra de palmas de chonta o de cultivos de yuca, por lo que existe una referencia constante de los waorani de "retornar" al espacio de los mayores "donde dejaron sembrando la chonta" u otros productos. Esta consideración de la selva como espacio doméstico explica la relación que mantienen con el entorno, como un espacio propio por los derechos otorgados por los antepasados ${ }^{10}$, permitiendo un manejo de amplias zonas donde se distribuían los onkos de los diferentes nanicabo a una distancia considerable, según la investigadora Laura Rival "de hasta dos días de camino" (1996: 24).

La forma de organización social permite la autosuficiencia de cada grupo familiar, requiriendo espacios de interrelación para el establecimiento de acciones de intercambio, entre ellas del matrimonio. Así, con el matrimonio fuera del grupo familiar se garantiza la exogamia, la sobrevivencia del grupo y se amplían las relaciones sociales que son elementos de alta importancia para los grupos familiares. Dichas prácticas se mantienen en los grupos familiares waorani en aislamiento donde el sistema de parentesco y sus normas permiten mantener y asegurar la cohesión grupal, mediante la circulación de bienes, entre ellos las mujeres, consolidando vínculos de consanguinidad y de alianza.

Los lazos de parentesco y alianzas establecidos a partir del intercambio de mujeres, permiten que el territorio compartido con otros grupo familiares sea un territorio de uso entre aliados, reduciendo la conflictividad que se podría presentar el momento de aprovechar los recursos naturales. Dichas alianzas garantizan la seguridad para el grupo, que podrían integrar a los individuos en acciones de defensa del territorio a través de ataques y de guerra. El parentesco y las reglas que surgen de un contexto cultural particular, aseguran la cohesión del grupo tanto hacia el interior, con la circulación de elementos materiales o simbólicos, como también hacia el exterior, en el caso waorani alianzas para la guerra, de esta manera se impulsa la circulación de bienes y consolida los vínculos de la consanguinidad. Las relaciones sociales y las dinámicas dentro de los grupos humanos se desarrollan en un contexto ambiental, y la circulación de elementos ya sean materiales, simbólicos o mensajes son expresiones de la cultura.

8 Onko es la construcción de aspecto triangular alargado que sirve como sitio de vivienda. El onko tiene unas dimensiones de aproximadamente 15 metros de largo por 6 de ancho, y una altura de hasta seis metros. Son seis pilares principales sobre los que se asientan travesaños que van a lo largo de la construcción y que sostienen a los maderos que de lado y lado se unen en el centro conformando el triángulo. Esta construcción tiene unos maderos livianos en función de correas donde se colocan las hojas que dan la forma característica a la casa waorani.

9 Si bien en épocas de paz manejan kewenkodes, donde siembran yuca y otros productos de horticultura básica, esta actividad es limitada por la constante movilidad a lo largo del territorio tradicional.

10 Sobre este tema, me encuentro en preparación de un extenso artículo donde se exponen varios aspectos sobre la relación con el entorno natural, el orden social y la conflictividad del territorio. 
En varias sociedades, las mujeres son consideradas un bien (Gómez García, 1981), por ser sujetas a un valor originado en su importancia en la reproducción social y biológica, y por tanto, son un elemento de intercambio. En el caso de las sociedades de filiación cultural waorani, las alianzas a través del matrimonio permitían acceder, al hombre, a los derechos territoriales sobre el territorio de los padres de la mujer, por tanto se ampliaba no solo la relación social y alianzas, sino la posibilidad de acceso a un territorio domesticado por los antepasados de línea femenina.

Además, el matrimonio y las relaciones que establece tiene una importancia en cuanto al valor que representa en alianzas para la paz y para la guerra; en la paz las alianzas permiten la ampliación del entorno familiar y social, la festividad y la posibilidad de continuar a lo largo del tiempo, y en la guerra representan la seguridad de integrar un grupo amplio para acciones de venganza o correrías de defensa.

\section{Dinámicas de intercambio e interrelación entre los grupos familiares en aislamiento y los waorani}

Los grupos familiares en aislamiento mantienen la forma de vida tradicional de los waorani antes del contacto, esto es una territorialidad basada en épocas de paz y épocas de guerra en un continuum de movilidad estacionaria (marcada por periodos relacionados con la fructificación de la chonta, del morete, algodón y otros, además de la cacería relacionada con esos periodos de fructificación) y cíclica (en la cual la movilidad se genera por los periodos de guerra donde se "retorna" al territorio de los ancestros por línea femenina). Además, los grupos familiares en aislamiento tienen un nivel de relaciones de intercambio de baja intensidad en su territorio de vida con pobladores waorani, tal como ha sido relatado por varios waorani.

- Yero: poblado de Ganketapade

- Ompore Omehuai: poblado de Yarentaro y sector de Ñmengono

- Manuel Cahuia: poblados de Ñoneno y Dicapare (Armadillo)

- Pego: poblado de Mihuaono

- Ocata: poblado de Yawepade

- Omayebe: poblado de Boanamo

- Kemperi: poblado de Baameno

Estos contactos de baja intensidad son esporádicos y se manifiestan en la circulación de bienes, como hachas, machetes, ollas y otros elementos que waorani han entregado a familias en aislamiento o que estas han "tomado" de poblados waorani o colonos. Las dinámicas de intercambio entre los grupos familiares en aislamiento y los waorani se expresan en el uso de un mismo territorio con ciertas fronteras culturales establecidas por los derechos otorgados por los antepasados que pueden vincular ciertos referentes pasados de parentesco común y donde existen recursos compartidos. Esos territorios de interrelación permiten una mutua existencia con el acceso a bienes esenciales como la cacería y recolección.

Es justamente ese tipo de relaciones de baja intensidad las que mantenía Ompore con individuos de grupos familiares en aislamiento, de las cuales tenían conocimiento sus familiares y otros waorani de diferentes poblados: "Ompore conversaba con Tagaeri, le iban a visitar a su casa y él iba a visitarles a su casa. Se encontraban también en la selva mientras iban a cacería, eso contaba que se encontraba" (Entrevista a Nemonca Ahua, mayo de 2013. Dicaro).

Las denominadas visitas podrían hacer referencia a encuentros en la selva en el territorio de interrelación e interdependencia, incluso algunas visitas que recibió Ompore en las cuales habría entregado hachas y machetes, y un compromiso de intercambio de elementos metálicos 
(por parte de Ompore) con lanzas (que serían entregadas por los aislados). De esta manera, la interrelación se establece por el desarrollo de actividades de recolección y cacería, de tránsito y de movilidad en dinámicas estacionarias y cíclicas en un mismo territorio; y, por otra de interdependencia porque los recursos se encuentran en la selva y de ellos dependen en el marco de su forma de vida.

\footnotetext{
"Ompore siempre estaba conversando con Taromenane, le pedían ollas, machetes, hachas. Último le habían pedido que saque a petrolera que hace mucho ruido. Le pidieron escopeta, Ompore no les había dado, les ofreció darles otro día. Taromenane le habían dicho que a cambio le entregaban lanza" (Entrevista a Ewenguime Enqueri, septiembre de 2013. Quito).
}

La información proporcionada en entrevistas realizadas a pobladores locales principalmente del grupo familiar de Ompore, expone un espacio de relacionamiento y de comunicación, donde se intercambia información, demandas y ofrecimientos, en códigos compartidos que evidencian ese origen cultural waorani y el manejo de la misma lengua ${ }^{11}$. Así mismo, un diálogo sostenido y reportado por un anciano waorani con un grupo familiar en aislamiento expresa el deseo de una época de paz, con relaciones que posibiliten potenciales intercambios. La época de paz manifiesta deseo de que este período se mantenga y se reafirme con la apertura de un canal para una visita pacífica y por tanto continuar con la territorialidad en un marco de estacionalidad; es decir, un acuerdo de época de paz: "Queremos que vivas bien. Vivamos bien. Tú en tu lugar, nosotros en el nuestro. Volveremos a visitar en otra ocasión" (CDES, 2012).

Las relaciones de intercambio se dan también entre varias familias waorani, que buscan mantener un espacio de circulación de bienes y recursos. Existen familias que preparan y mantienen chacras de yuca para que sean recolectadas por los aislados, estas prácticas se dan en poblados como Peneno, Guiyero, Yawepade, entre otros ${ }^{12}$. En esta dinámica de reciprocidad, los aislados dejan canastos con carne de monte ahumada. En esta expresión evidente de relación de intercambio tanto los grupos familiares en aislamiento como los waorani garantizan su persistencia tanto por el acceso a recursos para la subsistencia, como por la generación de acuerdos que reafirman la interdependencia sobre un territorio. "Por atrás de la casa vienen Tagaeri, se acercan a recoger yuca de las chacras, aquí dejamos nomas que cojan. Ellos dejan changuina ${ }^{13}$ de carne. Las huellas se encuentra, grandes los pies" (Entrevista a Yero, junio de 2013. Ganketapade).

Dentro de los elementos sujetos a intercambio entre waorani y grupos familiares en aislamiento, se encuentran los denominados "bienes esenciales" (Levi-Strauss, 1991), son los elementos principales para el intercambio los cuales permiten activar el principio de reciprocidad, y su valor es establecido por la relación entre la abundancia y escases de los mismos. En el caso de los grupos familiares en aislamiento, las mujeres y el territorio donde se recolectan los alimentos tienen la capacidad de conformar sistemas de relaciones reales y simbólicas en las dinámicas de intercambio al interior y entre grupos. En el caso de los grupos familiares en aislamiento las relaciones están marcadas tanto por las alianzas que se manejan a través del matrimonio entre grupos, como por intercambio de objetos, generalmente con waorani. Las herramientas de metal, como hachas y machetes, constituyen bienes de alta importancia para los pueblos en aislamiento, y sobre ellos se establece un valor que sobrepasa lo utilitario, ya que ha facilitado varias de las actividades cotidianas, como el derribo de árboles, confección de lanzas, entre otros.

11 Existe un mayor detalle en cuanto a los contenidos de la conversación que mantuvo Ompore con los aislados, narrados por él mismo, y que se encuentra en una entrevista en video (CDES, 2012).

12 Esta información se la obtuvo de varias entrevistas realizadas en dichos poblados entre 2013 y 2016.

13 Es el nombre kichwa dado a los canastos confeccionados con fibras naturales. 


\section{El ataque de un grupo familiar en aislamiento contra dos ancianos waorani}

El ataque contra los ancianos waorani Ompore y su esposa Buganei se produjo a primeras horas de la mañana del 5 de marzo de 2013, muy cerca al poblado de Yarentaro y después de que Ompore entregó varias piezas de cacería a su hermano Araba, quien las intercambiaría con un shaman que le estaba haciendo un tratamiento en su rodilla ${ }^{14}$.

"En una canasta Ompore había venido a dejar carne. Ese día había fiesta en la comunidad. Ya cuando dejó la carne había dicho: soñé mal, soñé bien malo, algo va a pasar. Él ya sabía que algo iba a pasar. La Buganei había empezado a decir que: la sanguijuela me ha estado chupando todo el cuerpo." (Entrevista a Orengo Tocari. 17 de junio 2013. Dicaro).

Ompore habría salido en la madrugada desde su casa ubicada en Ñemengono, a dos horas de Yarentaro, lugar donde habitaba desde hace varios años como una manera de alejarse del ruido de la empresa petrolera. Ompore era un anciano que retomó la forma de vida tradicional waorani, a pesar de que se incorporó al contacto en la década de los 70 (Labaka, 1988), esto es vivir de la cacería, recolección y el cultivo en los kewenkode ubicados cerca a su onko.

Una vez que hubo entregado la canasta a su hermano, y tomando el camino de regreso fue atacado por varios individuos de un grupo familiar en aislamiento. Buganei sobrevivió herida por algunas horas, tiempo en que pudo contar a sus familiares detalles del ataque, y afirmar que los autores fueron de familias en aislamiento. Los familiares de los ancianos pudieron evidencia algunos registros que quedaron del ataque, principalmente las lanzas ${ }^{15}$, además de las huellas de pisadas ${ }^{16}$ y el sendero utilizado para la emboscada y huida.

"De varias casa vinieron a atacar... La lanza que mató a Ompore es de viejo, un viejo vino" (Entrevista a Orengo Tocari, 14 de mayo 2013).

"Bastante gente estuvo en el ataque a Ompore, había mucha huella, pie grande donde mataron a Ompore" (Entrevista a Cawo Boya. 13 de mayo 2013. Peneno).

El grupo de atacantes habría estado conformado por varios individuos, en su mayoría jóvenes, destacándose la presencia de una persona mayor (en las entrevistas se le denomina como viejo). Un aspecto que se resalta es la conformación del grupo atacante la cual se caracterizó por la presencia de individuos de varios grupos familiares, lo que evidencia las alianzas existentes entre nanicabos en aislamiento ${ }^{17}$.

Los relatos de entrevistados indican distintas causas del ataque, una de ellas afirma la molestia de las familias en aislamiento por el ruido de las actividades en los pozos de la zona sur del bloque 16, que afectan a la cacería, y molestan la circulación por senderos de recolección y movilidad tradicional en esos territorios interdependientes con las familias waorani. Esta narración fue realizada por moradores de Dicaro y Yarentaro. "Advertencia a Ompore había sido unos cuatro meses antes, porque una de las familias en aislamiento había pedido ollas. Igualmente, le habían pedido que calle el ruido de los generadores, que traiga ollas y machetes" (Entrevista a Venancio Yeti. 14 de mayo de 2013. Dicaro).

14 Es frecuente que el pago por este tipo de servicios se de en productos o bienes. En el caso de Araba, acudía a un shaman kichwa del sector de Pompeya.

15 Al respecto, la Fiscalía solicitó dos peritajes de las lanzas que ocasionaron la muerte de los ancianos Ompore y Buganei, que corroboraron el origen de las lanzas (Rival, 2013; Narváez, 2013c).

16 Las huellas de pisadas de pueblos en aislamiento se caracterizan por evidenciar una forma de abanico, con los dedos de los pies separados.

17 Estas prácticas son conocidas entre las familias de habla wao tededo en épocas previas al contacto en la cual los atacantes organizaban al grupo familiar e invitaban a miembros de otras casas, de esta manera sellaban alianzas e integraban al grupo familiar: "Los grandes guerreros organizaban constantemente expediciones de guerra en contra de los blancos y los quichua, para lo cual necesitaban la ayuda de otros grupos" (Rival, 1996: 74). 
Como se mencionó, se conocía de relaciones de intercambio entre Ompore con alguna o algunas familias en aislamiento, a quienes había entregado algunos elementos como regalo, entre ellos ollas o machetes, y de allí se desprende una segunda causa que podría ser el fracaso en un intercambio que generó una ruptura en las relaciones y por tanto debilitó esa estrecha línea de comunicación, convirtiendo al potencial aliado en una amenaza.

En las primeras épocas del contacto de los waorani, en la década del 50 y 60, la incorporación de elementos externos a la cotidianidad de los waorani generó "conflictos internos" (Rival, 1996; 2001). Se señala puntualmente que el contacto con el exterior generó momentos de violencia intragrupal, principalmente para apropiarse de herramientas, como hachas, machetes u ollas; esta situación pudo y puede estar repitiéndose en el caso de las familias en aislamiento, disputas que generen conflictos y que provoquen rupturas de relaciones internas.

Otra información señala que en un encuentro de Ompore con grupos familiares en aislamiento ${ }^{18}$ se le había indicado que no debía realizar actividades de cacería en una zona que los PIA consideraban de su uso tradicional. En este caso, Ompore habría hecho caso omiso a dicha advertencia lo que desencadenó el ataque, más aún cuando el día del ataque habría salido con una canasta llena de carne silvestre.

El día del ataque, Buganey sobrevivió por varias horas, en este tiempo narró la conversación que mantuvieron los atacantes, que es narrada por sus familiares y donde se detalla el ingreso de un helicóptero sobre un onko de familias en aislamiento, sus moradores se habrían ocultado en la selva y pudieron ver luces que salían del helicóptero, posiblemente el flash de cámaras fotográficas; se señala, además, que bajó una cuerda $\left(\right.$ Cumi $\left.^{19}\right)$ y una red (Digindai $\left.{ }^{20}\right)$, con varios objetos que fueron depositados en el suelo.

\begin{abstract}
"[Un] helicóptero negro grandote aterrizó en chacra. Blanco, blanco salía. Siguiente día dejaron poco de cartones, atún abre fácil, al que llaman Deinemonta. Comieron, después dolió la barriga, a la semana han muerto" (Entrevista a Araba Omehuai, hermano de Ompore. 14 mayo 2013. Dicaro).

"Atacaron a Ompore porque pensaron que él fue a dejar la alimentación. Ellos vienen a botar (los helicópteros), comiendo se mueren." (Entrevista realizada y traducida por Ana Gaba a una de las niñas que fue capturada por los atacantes waorani en una incursión que realizaron como venganza a la muerte de Ompore y Buganei. 18 de junio 2013. Baameno).
\end{abstract}

Esta información proporcionada por una de las niñas expone la presión constante que existe sobre los grupos familiares en aislamiento, ya sea por sobrevuelos o entrega de "presentes" a través de distintos medios, cuyo uso y consumo puede generar situaciones de enfermedad o muerte. Estas acciones de actores externos evidencian un desorden en la administración pública de la región del Yasuní, a pesar de existir entidades a cargo del desarrollo e implementación de políticas públicas de protección a pueblos en aislamiento ${ }^{21}$.

En los waorani previo al contacto se conoce que las causas para realizar las correrías o ataques tenían relación con matar para vengar a sus muertos, para defender el territorio y para acceder a bienes del exterior como herramientas, sin embargo, si hubo una muerte al interior de un grupo de familias en aislamiento, esto pudo haber incidido en el ataque: "Ellos mataban para vengar sus muertos, para tomar represalias contra los invasores, y para robar sus herramientas" (Rival, 1996: 74).

18 Esta información fue recogida de una entrevista realizada a varios miembros de la familia, entre ellos su hijo Cawime en mayo de 2013.

19 Piola o cuerda de chambira utilizada por los hombres para sostener el pene.

20 Es una bolsa tejida de chambira.

21 Desde 1996 el Estado ecuatoriano cuenta con una entidad a cargo de la protección de los pueblos en aislamiento, inicialmente estuvo inserta en la estructura del Ministerio del Ambiente como "Plan de Medidas Cautelares para la protección de los pueblos en aislamiento voluntario Tagaeri Taromenane”, y que pasó, posteriormente al Ministerio de Justicia, Derechos Humanos y Cultos y hoy se maneja como Dirección de Promoción de Pueblos Indígenas en Aislamiento Voluntario. 
En este contexto, la información existente da pie a múltiples versiones en torno a las razones del ataque; sin embargo, no se puede tomar una sola vía de interpretación y se debe sostener que el evento de lanceamiento de los ancianos Ompore y Buganei pudo tener múltiples orígenes. El análisis que se busca establecer es que dentro de todo el contexto de diversidad de actores presentes en la región del Yasuní, que es territorio de los waorani como pueblo de reciente contacto y varios grupos familiares en aislamiento waorani, es claro que existen relaciones de intercambio entre familias de pueblos en aislamiento y pobladores waorani, las cuales por distintas circunstancias pudieron haberse roto, ya sea por presiones externas o dinámicas propias al interior del territorio, como el incremento en la cacería, presión sobre un territorio considerado propio por las familias en aislamiento, muerte de algún miembros de familias en aislamiento desencadenando acciones de venganza, y otras sobre las que es necesario profundizar en investigación.

Si bien estas relaciones entre waorani y grupos familiares en aislamiento evidencian un estado de paz y acuerdos explícitos sobre un territorio que comparten, y que se expresan en una circulación de bienes con una serie de características, como ser exóticos, especiales, externos, no comunes, vistosos, con un valor que puede ser incluso utilitario, y por eso son bienes importantes. La eficiencia de los bienes es también importante, pues una herramienta metálica permite mejores resultados que una de piedra o de madera de chonta.

Es así que con la información expuesta, se identifican dinámicas que pudieron haberse roto, impidiendo las relaciones de intercambio e interacción esporádicas y de baja intensidad y la relación pacífica sobre un mismo espacio territorial de interdependencia. La ruptura en el acceso a elementos del exterior limitó un círculo de relación, que pudo incidir en el ataque contra los ancianos waorani. Es importante tener presente que los pueblos en aislamiento, a través de contactos de bajo nivel o esporádicos, buscarían mantener un medio para intercambio, sobre todo para acceder a productos que les hacen faltan. Es preciso destacar que los elementos que recibían los aislados por parte de waorani tienen mucho valor utilitario al igual que simbólico, estos pudieron haber sido considerado como elementos exóticos, vistosos, especiales, no comunes, y eficientes. La relaciones existentes al ser rotas o al no poder consolidarse exponen una imposibilidad de establecer una alianza, que en un espacio territorial donde la interrelación era una constante dejaba al aire una posible amenaza al estar compartiendo un territorio con enemigo.

De esta manera, relaciones de intercambio que no se concretaron dieron paso a relaciones hostiles que resumen el sentido del intercambio como la manifestación de una "guerra resuelta de forma pacífica" (Levi-Strauss, 1991), con el consiguiente resultado de que una relación frustrada da paso a la guerra o a la violencia, como expresión de esa transacción desafortunada.

\section{Conclusiones}

La circulación de bienes entre waorani y familias de pueblos en aislamiento implica una dinámica de relación en un territorio compartido donde existe una interrelación constante e interdependencia de sus recursos. Las relaciones existentes permiten el uso y la movilidad en una expresión de territorialidad, donde la relación activa asegura un periodo de paz. De esta manera, se mantendrían y se mantienen latentes en el marco de alianzas y rivalidades. Si bien el matrimonio es una puerta a círculos de intercambio o los consolida, no existen datos certeros que expongan la circulación de mujeres como un factor que pudo haber desencadenado el ataque.

Existen espacios y tiempos de contacto entre waorani y grupos familiares en aislamiento, en los cuales se han establecido ciertas condiciones de intercambio, permitiendo a los aislados acceder a productos que les faltan, como las herramientas metálicas, ollas y otros implementos. Las relaciones de intercambio constituyen un continum entre las relaciones hostiles y las prestaciones o donaciones recíprocas, siendo éstas el uso de un territorio, el acceso a recursos de la selva y la garantía de un tiempo de paz. 
El ataque con lanzas que sufrieron los ancianos waorani Ompore y Buganei aparentemente tuvo varias causas, entre ellas una relación de intercambio que no pudo sostener las dinámicas de interrelación en el territorio compartico con familias en aislamiento, donde existe una interdependencia de los limitados recursos que allí existen, principalmente por la presión de actores externos que limitan tanto una circulación por el territorio tradicional e inciden en la abundancia de fauna por sobrevuelos y ruidos de generadores, entre otros. Así, el ataque de un grupo de familias en aislamiento contra Ompore y Buganei deja claro la fragilidad en las relaciones existentes en el territorio tradicional waorani, donde la presión sobre los recursos naturales y el área de territorialidad en la que se desenvuelven los grupos familiares en aislamiento en el Yasuní desencadena situaciones de violencia.

Es evidente además, que la presión existente desde los actores externos que se desenvuelven en el mismo territorio, como empresas petroleras, frontera de colonización, turismo desordenado, y otras, inciden en hechos de violencia, como el sucedido en marzo de 2013 en el poblado de Yarentaro, y ante lo cual se requiere profundizar las investigaciones en el ámbito social y cultural para el desarrollo de políticas públicas acorde a las dinámicas existentes al interior del territorio, donde los grupos familiares en aislamiento constituyen una prioridad para la implementación de garantías de protección desde el Estado.

\section{Bibliografía}

Cabodevilla, Miguel Ángel. 1999, Los huaorani en la historia de los pueblos del Oriente, CICAME, Coca.

CDES, Ompore: me dijeron los Taromenani, tomado de https://www.youtube.com/watch?v=qcYpj9cOrI8, (30 de enero de 2012).

Cerón, Carlos, \& Montalvo, C. 1998, Etnobotánica de los Huaorani de Quehueri - Ono, Abya-Yala, Quito.

Cipolleti, M. 2002, "El testimoio de Joaquina Grefa, una cautiva quichua entre los waorani" (Ecuador, 1945). Journal de la Societe des Americanistes (En Ligne).

Gómez García, P. 1981, La antropología estructural de Claude Lévi-Strauss, Editorial Tecnos, Madrid.

Labaka, A. 1988, Crónica Huaorani, CICAME, Coca.

Levi-Strauss, C. 1991, Las estructuras elementales del parentesco, Ediciones Paidós, Barcelona.

Narváez, R. 2014, Estudio socio poblacional del Territorio Sapara. Terra Mater, Quito.

2013 ${ }^{\text {a }}$ Estudio de Antropología Jurídica sobre los hechos ocurridos en el Yasuní, en el poblado de Yarentaro, Informe Técnico Sin Públicar, Fiscalía General del Estado. Comisión de la Verdad y Derechos Humanos, Quito.

2013b, Alcance al Estudio de Antropología Jurídica. Informe técnico sin publicar, Fiscalía General del Estado. Comisión de la Verdad y Derechos Humanos, Quito.

2013c, "Análisis comparativo de lanzas Tagaeri Taromenane que han intervenido en muertes y ataques", Fiscalía General del Estado. Comisión de la Verdad y Derechos Humanos, Quito.

Oficina del Alto Comisionado de Naciones Unidas para Derechos Humanos. 2012, Directrices de protección para los pueblos indígenas en aislamiento y en contacto inicial de la Región Amazónica, el Gran Chaco y la región oriental de Paraguay, OACNUDH, Ginebra.

Rival, L. 2013, Informe de peritaje de lanzas de Yarentaro, Fiscalía de Orellana, Coca.

1996, Hijos del sol padres del Jaguar, Abya-Yala, Quito.

Rivas, A. 2003, "Sistema mundial y pueblos indígenas en la Amazonía: a propósito del ataque a los tagaeri (Coyuntura)", en: ICONOS, Revista de ciencias sociales, No. 17, FLACSO sede Ecuador, pp. 21 - 30.

Rivas, A. 2006, Los pueblos indigenas en aislamiento desde los derechos humanos y la conservación de la biodiversidad, texto para la Octava Conferencia de las Partes, Convenio de Diversidad Biológica, Curitiba, Brasil.

Tirdmarsh, W. 1945, Unarranged Notes on the Aucas of the Nushiño Basin, Mecanografiado.

Yost, J. 1978, El desarrollo Comunitario y la Supervivencia Étnica. El caso de los huaorani, Amazonía Ecuatoriana, Instituto Lingüístico de Verano - Ministerio de Educación y Cultura del Ecuador, Quito. 